\title{
Questes
}

\section{Les mouches blanches, qui piquent-elles? Rutebeuf sous la neige avec les Ribauds de Grève}

\section{Sung-Wook Moon}

\section{(2) OpenEdition}

Journals

\section{Édition électronique}

URL : http://journals.openedition.org/questes/4378

DOI : 10.4000/questes.4378

ISSN : 2109-9472

\section{Éditeur}

Les Amis de Questes

\section{Édition imprimée}

Date de publication : 15 décembre 2016

Pagination : 55-84

ISSN : 2102-7188

\section{Référence électronique}

Sung-Wook Moon, «Les mouches blanches, qui piquent-elles ? Rutebeuf sous la neige avec les

Ribauds de Grève ", Questes [En ligne], 34 | 2016, mis en ligne le 21 décembre 2016, consulté le 30 avril 2019. URL : http://journals.openedition.org/questes/4378 ; DOI : 10.4000/questes.4378 


\title{
Les mouches blanches, qui piquent-elles ? Rutebeuf sous la neige avec les Ribauds de Grève
}

\author{
Sung-Wook MOON \\ Université Paris-Sorbonne
}

\begin{abstract}
Rutebeuf est un grand polygraphe. À travers sa cinquantaine de poèmes, ce rimeur $\mathrm{du} \mathrm{XIII}^{\mathrm{e}}$ siècle joue de registres variés, allant de l'hagiographie au fabliau. Mais sa renommée est essentiellement due à onze textes, réunis par Edmond Faral et Julia Bastin sous le nom fameux de Poèmes de l'infortune ${ }^{1}$. Notre but, très modeste, est d'analyser une pièce de ce groupe, le Dit des ribauds de Grève. Toutefois, il nous faut au préalable faire un détour par les Poèmes de l'infortune dans leur ensemble en nous attachant à leur tradition éditoriale. Puis nous pourrons opérer une comparaison entre ce poème et d'autres textes de Rutebeuf ou de ses contemporains. Cette démarche, nous l'espérons, démontrera à la fois la singularité de la pièce en question et son caractère révélateur du travail entier de cet auteur.
\end{abstract}

\section{Les Ribauds de Grève : un poème de l'infortune?}

Si, pour nous modernes, les Poèmes de l'infortune marquent le sommet de son œuvre, l'infortune qu'y dépeint Rutebeuf n'est pas tout à fait originale ni forcément authentique. Depuis l'ouvrage pionnier de

\footnotetext{
${ }^{1}$ CEuvres complètes de Rutebeuf, éd. Edmond Faral et Julia Bastin, Paris, Picard, 1959-1960, 2 vol., t. 1, p. 517-580.
} 
Nancy Freeman Regalado ${ }^{2}$, la critique moderne y voit moins des témoignages sincères que des lieux communs hérités. Mais cette infortune a au moins une spécificité, celle de relever d'une expérience que le poète prétend avoir vécue. C'est lui-même qui aurait été affligé par la pauvreté et la maladie, et qui en outre n'aurait cessé d'aggraver ces malheurs par ses multiples travers: le vin, le jeu de dés et un mariage déraisonnable avec une femme dépourvue et de beauté et de richesse, mais qui avait «cinquante anz $[\ldots]$ en son escuele ${ }^{3} »$. En somme, à l'en croire, sa peine dépasserait même celle des saints martyrs :

C'il ont estei por Dieu deffait, Rosti, lapidei ou detrait, Je n'en dout mie, Car lor poinne fu tost fenie, Et ce duerra toute ma vie Sanz avoir aise ${ }^{4}$.

Cette vie de souffrance connaît néanmoins des variations qui suivent en grande partie le rythme naturel des saisons. L'été offre une trêve aux tourments des misérables de son genre :

Et avris entre,

Et il n'ont riens defors le ventre.

Lors sunt il vite et prunte et entre,

Eiz vos la joie ! [...]

Or lor at Dieux un tenz prestei

\footnotetext{
${ }^{2}$ Nancy Freeman Regalado, Poetic Patterns in Rutebeuf: A Study in Noncourtly Poetic Modes of the Thirteenth Century, New Haven/Londres, Yale University Press, 1970.

3 Rutebeuf, Mariage Rutebeuf, v. 36, dans Euvres complètes, éd. et trad. Michel Zink, éd. revue et mise à jour, Paris, Le Livre de Poche, 2001 [1989_ 1990], p. 270 (nous renverrons désormais à cette édition de référence par le sigle « $\mathrm{MZ} »)$. Ce trio de vices est conforme à l'idée répandue à l'époque, telle que la résume le proverbe suivant : "Par vin, par fame et par dez Si vient toust homme a povretez » (Proverbes français antérieurs au XV ${ }^{e}$ siècle, éd. Joseph Morawski, Paris, Champion, 1925, p. 58, $\mathrm{n}^{\circ} 1603$ ). Pour la manière dont Rutebeuf amplifie ce topique, voir Nancy Freeman Regalado, Poetic Patterns in Rutebeuf, op. cit., p. 286-288.

${ }^{4}$ Rutebeuf, Mariage Rutebeuf, v. 128-133, dans MZ, éd. cit., p. 276.
} 
Ou il fait chaut,

Et d'autre choze ne lor chaut :

Tuit apris sunt d'aleir deschauz .

En revanche, l'hiver fait que leur misère éclate sous la violence du froid :

Povre sens et povre memoire

M'a Diex donei, li rois de gloire,

Et povre rente,

Et froit au cul quant byze vente :

Li vens me vient, li vens m'esvente

Et trop souvent

Plusors foïes sent le vent ${ }^{6}$.

Entre l'hiver et l'été, marqués respectivement par un crescendo et un decrescendo de la douleur, la vie du pauvre obtient-elle finalement, par quelque effet de providence, un bilan équilibré ? C'est loin d'être le cas. L'hiver, après tout, l'emporte sur la chaleur estivale par sa propre force glaciale: « Ne voi venir avril ne mai, / Veiz ci la glace $^{7} »$. En outre, quand il vient, l'été n'apporte pas grand-chose, car, privé de vêtement, le pauvre n'échappe jamais au froid. Là réside la puissance du jeu de dés, la griesche qui

Juignet li fait sembleir fevrier :

La dent dit : «Quac»,

Et la griesche dit : «Eschac».

Qui plus en set s'afuble .I. sac

De la griesche ${ }^{8}$.

Voilà le paysage dominant des Poèmes de l'infortune, espace gelé et dévasté où le poète est enfoncé, et qui devient encore plus insupportable quand le vent farouche se lève, au point d'emporter ses amis qui devraient lui donner la main :

Se sont ami que vens enporte,

\footnotetext{
5 Rutebeuf, Griesche d'été, v. 105-108 et 113-116, dans MZ, éd. cit., p. 210.

6 Rutebeuf, Griesche d'hiver, v. 10-16, dans MZ, éd. cit., p. 196.

7 Ibid., v. 59-60, p. 198.

${ }^{8}$ Rutebeuf, Griesche d'été, v. 20-24, dans MZ, éd. cit., p. 204.
} 
Et il ventoit devant ma porte,

Ces enporta,

C'onques nuns ne m'en conforta

$\mathrm{Ne}$ riens dou sien ne m'aporta ${ }^{9}$.

Pourtant, les Poèmes de l'infortune ne constituent pas un bloc homogène, mais comportent quelques pièces de caractère différent : le Dit de Renart le Bestourné, poème polémique qui met en cause les frères mendiants et leur protecteur, le roi de France saint Louis; le Dit d'Aristote qui, s'appuyant sur l'autorité du philosophe antique, préconise les vertus dignes du prince, au premier rang desquelles la largesse ; De Brichemer, brocard lancé à un seigneur qui ne tient pas la promesse de don matériel ; et, enfin, le Dit des ribauds de Grève, objet de notre article. Mais, avant d'engager la lecture de celui-ci, nous nous permettrons quelques mots de plus sur l'ensemble des Poèmes de l'infortune et la longue gestation de ce regroupement, dont l'hétérogénéité apparente s'explique par l'histoire des éditions consacrées à Rutebeuf depuis le XIX ${ }^{\mathrm{e}}$ siècle.

En 1839, lors de la première publication des Euvres complètes du poète, Achille Jubinal a réparti le corpus en cinq catégories.

Dans l'impossibilité où nous étions de leur assigner une place chronologique, puisque rien ne faisait reconnaître pour plusieurs la date de leur composition, nous avons compris dans notre premier volume: $1^{\circ}$ les pièces composées par Rutebeuf sur lui-même, $2^{\circ}$ les pièces relatives à de grands personnages et à de grands événements, $3^{\circ}$ les pièces satiriques, $4^{\circ}$ les fabliaux et contes. Quant aux poésies pieuses et allégoriques, au drame religieux et aux vies de saintes [...] nous en avons composé notre deuxième volume ${ }^{10}$.

\footnotetext{
${ }_{9}$ Rutebeuf, Complainte Rutebeuf, v. 122-126, dans MZ, éd. cit., p. 324.

${ }^{10}$ Euvres complètes de Rutebeuf, trouvère du XIII siècle, éd. Achille Jubinal, Paris, Pannier, 1839, 2 vol., t. 1, p. XXIX.
} 
Le premier groupe d'Achille Jubinal se constitue de sept pièces : Pauvreté Rutebeuf; Mariage Rutebeuf; Complainte Rutebeuf; Paix de Rutebeuf; Griesche d'hiver; Griesche d'été; Mort (ou Repentance) Rutebeuf, tandis que les Ribauds de Grève se trouvent dans le troisième, parmi les satires. Dans ce classement qui survivra à la révision de 1874 se manifeste clairement le prestige accordé aux sept poèmes occupant les places de tête ${ }^{11}$ et qui, en 1938, feront objet d'une publication indépendante que donnera Harry Lucas sous le titre parlant: Les Poésies Personnelles ${ }^{12}$.

11 Euvres complètes de Rutebeuf, trouvère du XIII siècle, éd. Achille Jubinal, nouvelle éd. revue et corrigée, Paris, Paul Daffis, 1874-1875, 3 vol. Sur ce point, l'édition d'Adolf Kressner, publiée en 1885, ne semble pas changer beaucoup, malgré quelques modifications de détail dans l'ordre des textes (Rustebuef Gedichte. Nach den Handschriften der Pariser National-Bibliothek, éd. Adolf Kressner, Wolfenbüttel, Zwissler, 1885). Mais la critique du XIX ${ }^{\mathrm{e}}$ siècle n'a pas toujours respecté la décision de ces éditeurs. Trois ans après la première édition d'Achille Jubinal, Paulin Paris adoptait pour son travail une division tripartite: jonglerie ; enseignements moraux - éloges - satires ; poésies pieuses. Il répartissait les poèmes selon ses propres convictions, en donnant la présentation de chacun. Mais dans sa liste ne figuraient pas quelques pièces, dont les Ribauds de Grève (Paulin Paris, "Rutebeuf», dans Histoire littéraire de la France, t. 20, Suite du treizième siècle, depuis l'année 1286, Paris, Didot Frères-Treuttel et Wurtz, 1842, p. 719-798). Quelques décennies plus tard, postérieurement donc à la deuxième édition d'Achille Jubinal et à celle d'Adolf Kressner, l'ouvrage synthétique de Léon Clédat réservait cinq chapitres à la présentation des œuvres, respectivement consacrés aux pièces lyriques, aux pièces satiriques, aux poèmes allégoriques, aux œuvres dramatiques et aux vies de saints et fabliaux, avec trois chapitres introductifs et une conclusion. Les «satires personnelles » étaient incorporées aux satires en général, mais étant donné que la plupart d'entre elles avaient été déjà amplement traitées au deuxième chapitre, «Vie de Rutebeuf », l'auteur s'est limité à faire lire deux autres textes : le Dit des Ribauds de Grève et la Dispute de Charlot et du Barbier de Melun, dont le premier donne à ses yeux, par anticipation, «la même note » que les poèmes villoniens (Léon Clédat, Rutebeuf, Paris, Hachette, 1891, en particulier p. 131-133).

12 Rutebeuf, Les Poésies Personnelles, éd. Harry Lucas, Genève, Slatkine Reprints, 1974 [1938]. Cette tradition plus que centenaire, colorée d'un certain romantisme, sera couronnée par l'article de Gustave Cohen : "Rutebeuf, l'ancêtre des poètes maudits », Études classiques, vol. 21, 1953, p. 1-18. Pour une réflexion approfondie sur ce sujet, voir Michel Zink, «Poète sacré, poète maudit », dans Modernité au Moyen Âge, dir. Brigitte Cazelles et Charles Méla, Genève, Droz, 1990, p. 233-247 ; Alain Corbellari, «De Rutebeuf à Léo Ferré : les fortunes du "poète maudit" ", dans Réception du Moyen Âge dans la culture moderne, 
En 1959-1960, cette organisation traditionnelle a été sérieusement revue dans l'édition d'Edmond Faral et Julia Bastin. Sans a priori chercher à proposer un principe radicalement différent, les deux éditeurs ont su s'appuyer sur les progrès de l'histoire et de la philologie pour donner une nouvelle répartition, toujours en cinq parties, cependant selon des critères plus précis et plus convaincants : L'Église, les Ordres mendiants et l'Université; Les Croisades; Poèmes de l'infortune; Poèmes religieux; Pièces à rire. De plus, à l'intérieur de chaque partie, ils ont tenté dans la mesure du possible d'ordonner les pièces suivant la chronologie ${ }^{13}$, ce qui avait été considéré comme inenvisageable par Achille Jubinal.

Dans ce système refondu, les poésies personnelles, fraîchement baptisées Poèmes de l'infortune, se sont enrichies de quatre pièces, et les philologues n'ont pas négligé de justifier au moins implicitement ces déplacements. Leur principal argument repose avant tout sur l'expression d'un souci matériel, que ce sentiment se cache sous un conseil de générosité, comme dans le Dit d'Aristote, ou à l'inverse, sous la satire contre un avare, comme dans Renart le Bestourné ou De Brichemer. Ces trois pièces semblent dès lors avoir bien le droit de côtoyer les poésies personnelles proprement dites. Toutefois cela ne peut être le cas des Ribauds de Grève:

Ribaut, or estes vos a point :

$\mathrm{Li}$ aubre despoillent lor branches

Et vos n'aveiz de robe point, Si en aureiz froit a voz hanches. Queil vos fussent or li porpoint Et li seurquot forrei a manches ! Vos aleiz en etei si joint,

dir. Danielle Buschinger, Amiens, Presses du Centre d'études médiévales, 2002, p. 52-60.

${ }_{13}$ Voir CEuvres complètes de Rutebeuf, éd. Edmond Faral et Julia Bastin, éd. cit., t. 1, p. 220-221. 
Et en yver aleiz si cranche!

Vostre soleir n'ont mestier d'oint :

Vos faites de vos talons planches.

Les noires mouches vos ont point,

Or vos repoinderont les blanches ${ }^{14}$.

Dans une certaine mesure, ces douze vers s'intègrent à bon droit parmi les Poèmes de l'infortune, et cela sur la base non pas d'un implicite conjecturé, mais de ce qui est effectivement inscrit dans le texte: après le premier vers ironique résumant l'état des ribauds, chaque phrase, chaque motif rappellent tel ou tel passage des poésies personnelles, notamment des deux Griesches. Ainsi le deuxième vers, où le poète évoque les arbres dépouillés en hiver, rappelle-t-il un autre début hivernal, celui de la Griesche d'hiver :

Contre le tenz qu'aubres deffuelle,

Qu'il ne remaint en branche fuelle

Qui n'aut a terre $[\ldots]^{15}$.

De même, le manque de vêtements et de chaussures, le comportement variable selon le temps, sont des thèmes que l'on a pu voir ci-dessus dans les exemples tirés des Griesches, hivernale et estivale. Reste que les Ribauds de Grève se présentent comme un tableau de froidure où le souvenir de la chaleur n'est plus qu'un repoussoir au présent douloureux. Ce n'est donc pas par hasard si le poème finit par deux vers qui nous ramènent à un tercet de la Griesche d'hiver:

Diex me fait le tens si a point,

${ }_{14}$ Rutebeuf, Dit des ribauds de Grève, v. 1-12, dans MZ, éd. cit., p. 214.

15 Rutebeuf, Griesche d'hiver, v. 1-3, dans MZ, éd. cit., p. 196. Même saison et même motif pour l'incipit du Mariage Rutebeuf: «En l'an de l'Incarnation, / VIII. jors aprés la Nacion / Celui qui soffri passion, / En l'an sexante, / Qu'abres ne fuelle, oizel ne chante [...]» (v. 1-5, dans MZ, éd. cit., p. 268). On sait d'ailleurs que le procédé est déjà bien établi et souvent pratiqué par la poésie courtoise, à côté de son pendant qui est autrement plus répandu, à savoir le début printanier. Voir Roger Dragonetti, La Technique poétique des trouvères dans la chanson courtoise. Contribution à l'étude de la rhétorique médiévale, Genève, Slatkine Reprints, 1979 [1960], p. 169-193. 
Noire mouche en estei me point, En yver blanche ${ }^{16}$.

Sur la base de cette confrontation, il est facile de comprendre pourquoi Edmond Faral et Julia Bastin ont placé les Ribauds de Grève parmi les Poèmes de l'infortune, juste après les Griesches ${ }^{17}$. Il n'empêche, cette décision est discutable. En dépit de similitudes évidentes, la moquerie contre les ribauds est d'une nature différente de l'autodérision caractéristique des poésies personnelles, car le poète ne dit pas ici un seul mot de lui-même, mais focalise sa vision exclusivement sur les ribauds, qu'il désigne par vos, à la deuxième personne du pluriel. En conséquence, la répétition de l'image entomologique que l'on vient de souligner explicite en réalité l'écart entre ses deux occurrences: d'un côté, le poète se plaint du harcèlement des mouches - noires et blanches, à savoir de vrais insectes et des flocons de neige ${ }^{18}-$ et de l'autre, c'est sur les ribauds

16 Rutebeuf, Griesche d'hiver, v. 31-33, dans MZ, éd. cit., p. 198.

17 Il en est de même pour l'édition de Michel Zink, qui refuse néanmoins tout groupement thématique et applique une chronologie hypothétique à la mise en ordre des textes. Ce n'était pourtant pas, on l'a vu, la place habituelle du poème jusqu'à la première moitié du $\mathrm{XX}^{\mathrm{e}}$ siècle, pas plus que celle du Moyen Âge : le seul manuscrit conservant ce dit (Paris, BnF, ms. fr. 1635, fol. 44v) le place entre le Dit des propriétés de Notre Dame (alias les Neufs joies de Notre Dame), aujourd'hui communément rejeté du corpus de Rutebeuf, et la Pauvreté Rutebeuf, tandis que les deux Griesches, liées l'une à l'autre, se trouvent plus loin (fol. 52r-54r). Notons que ce recueil, dont la logique d'organisation n'est généralement pas très facile à comprendre, a tout de même pris soin d'associer aussi le Mariage et la Complainte Rutebeuf (fol. 47r-49r), deux poèmes qui forment une autre paire. Pour la liste des textes de ce manuscrit, voir la notice de la Section Romane dans la base JonasIRHT/CNRS, site internet: http://jonas.irht.cnrs.fr/manuscrit/45638 (page consultée le 26 août 2015). Sylvia Huot en a offert une brève mais précieuse analyse dans son ouvrage From Song to Book. The Poetics of Writing in Old French Lyric and Lyrical Narrative Poetry, Ithaca/Londres, Cornell University Press, 1987, p. 215-216.

${ }^{18}$ Notons en passant que cette image paraissait hermétique aux yeux du premier éditeur du poète qui a écrit, en hésitant : «Le sens de cette pièce [est] assez difficile à comprendre [...] Par les noires mouches, je crois qu'il faut entendre : les puces, qui viennent surtout durant l'été, et par les blanches... le dirai-je ?... les poux. Hors de ces deux sens, assez peu nobles, j'en conviens, je ne vois pas ce que pourraient signifier les deux derniers vers du Diz des Ribaux de Greive, non plus que ceux sur 
que se jettent les deux espèces de mouches, tandis que le je reste observateur, ne partageant apparemment pas les tourments de ceux auxquels il s'adresse. Entre vos et je, la distance fait écho à celle qui sépare de la plainte subjective la moquerie prenant autrui pour cible. Il faudrait donc reconnaître là, avec Alain Corbellari, «le meilleur argument à opposer aux partisans d'un Rutebeuf poète "personnel"19 », et opter pour un autre Rutebeuf, expert en répétitions, topoï et locutions figées, profil vigoureusement promu par Nancy Freeman Regalado.

Admettons qu'il est difficile d'adhérer à l'opinion d'Edmond Faral :

C'est sans doute le sentiment de sa propre misère qui lui a inspiré sur le sort pitoyable de la plèbe des tavernes, des ribauds de la place de Grève, tous gens perdus par le vice, la paresse ou les duretés de la société, quelquesuns des vers les plus émouvants de notre littérature ${ }^{20}$.

Si l'on considère le poème indépendamment des autoportraits du poète, le ton des Ribauds de Grève trahit plutôt un sentiment de mépris que de pitié. Cette impression est renforcée par le fait que, comme l'a remarqué Nancy Freeman Regalado, Rutebeuf se contente alors de décrire la situation extérieure de la troupe des infortunés sans se soucier

le même sujet qui se trouvent [...] dans la pièce intitulée : De la Griesche d'yver » (CEuvres complètes de Rutebeuf, éd. Achille Jubinal, 1839, éd. cit., t. 1, p. 211). Le mot honteux de «poux » le gênait tellement qu'il l'a remplacé, dans sa deuxième édition, par un terme moins précis : «un autre genre de vermine » (CEuvres complètes de Rutebeuf, éd. Achille Jubinal, 1874-1875, éd. cit, t. 2, p. 7). Ce qui ne manquera pas de faire sourire Edmond Faral, qui écrira : «Il est superflu de relever les erreurs de Jubinal pour l'interprétation de cette pièce, dont il a trouvé le sens "assez difficile" » (Euvres complètes de Rutebeuf, éd. Edmond Faral et Julia Bastin, éd. cit., t. 1, p. 531).

19 Alain Corbellari, La Voix des clercs. Littérature et savoir universitaire autour des dits du XIII ${ }^{e}$ siècle, Genève, Droz, 2005, p. 161-162.

${ }^{20}$ Euvres complètes de Rutebeuf, éd. Edmond Faral et Julia Bastin, éd. cit., t. I, p. 45. 
de la cause ou de la signification morale de cette situation ${ }^{21}$, ce qui ne va pas dans le sens de la compréhension ou de la compassion qu'il aurait pu éprouver. Bien au contraire, le terme peu flatteur de ribaud, dont ces pauvres sont affublés dès le premier vers, suggère selon Michel Zink l'association entre «misère et débauche », si présente dans la tête des médiévaux que ces derniers peuvent en déduire que de tels êtres ne sont tourmentés que par leurs propres fautes ${ }^{22}$.

En réalité, l'ensemble et le titre des Poèmes de l'infortune sont des inventions de la philologie moderne, et les Ribauds de Grève s'en distinguent nettement ainsi que nous l'avons montré. Toutefois, en dépit de leurs différences fondamentales, des coïncidences visibles subsistent encore, notamment l'image des mouches. Bien entendu, ces insectes n'ont pas à chaque fois la même visée, se lançant à l'assaut tantôt des ribauds, tantôt du je lui-même. Mais l'image elle-même reste identique et revient dans les deux cas sous une formule semblable. Et elle paraît tellement inoubliable qu'il est légitime de supposer qu'en la répétant à bon escient, l'auteur ait voulu produire un effet de réminiscence ${ }^{23}$.

Certes, l'attachement de la littérature médiévale à la répétition est connu $^{24}$. Cela ne veut pourtant pas dire que ce procédé soit anodin et insignifiant. Au contraire, il consiste à exploiter la force et le sens de

${ }^{21}$ Nancy Freeman Regalado, Poetic Patterns in Rutebeuf, op. cit., p. 308-309. Voir aussi, du même auteur, "Two Poets of the medieval city », Yale French Studies, vol. 32, Paris in Literature, 1964, p. 12-21, en particulier p. 15-16.

${ }^{22}$ MZ, éd. cit, p. 214-215. Sur la liaison supposée de la pauvreté et des vices de ses victimes, voir Michel Mollat, Les Pauvres au Moyen Âge, Paris, Complexe, 2006 [1978], p. 92-97 et 129-142 ; Bronislaw Geremek, La Potence ou la pitié. L'Europe et les pauvres du Moyen Âge à nos jours, trad. Joanna Arnold-Moricet, Paris, Gallimard, 1987 [1978], p. 27-51.

23 De cette figure, aucune autre occurrence que ces deux n'est relevée par Adolf Tobler et Erhard Lommatzsch, Altfranzösisches Wörterbuch, Wiesbaden/Stuttgart, Steiner, 1925-1995, 11 vol., t. 6, p. 318.

${ }^{24}$ Cet attachement se trouve élevé au rang de particularité esthétique de l'époque par le livre majeur de Paul Zumthor : Essai de poétique médiévale, Paris, Le Seuil, 2000 [1972]. 
l'élément mis en œuvre, surdéterminé chaque fois par le contexte. Les agents - producteurs, diffuseurs ou récepteurs - de cette littérature étaient conscients de ce mécanisme, si bien qu'ils pouvaient à partir de là jouer à un jeu subtil de ressemblances et de dissemblances. Daniel Poirion a postulé il y a une trentaine d'années, s'agissant de la mouvance des textes alimentée par les copistes, que «les variations apportées à un texte par des variantes de détail ou des versions nettement divergentes ont elles-mêmes un sens et une histoire qu'il faut retrouver ${ }^{25} »$. Ce principe vaut à plus forte raison pour les variations produites par le poète lui-même, variations dont nous allons tenter de saisir, à partir de notre exemple, quelques aspects qui méritent notre attention.

\section{Jeu dialogique sans dialogue}

Concernant les Ribauds de Grève, ce qui rend particulière la scène typique de la froidure et du dénuement est avant tout l'usage constant de la deuxième personne du pluriel ${ }^{26}$. Bien sûr, ce n'est pas l'apanage de cette pièce. L'auteur y a recours dans plusieurs poèmes, d'une façon ou d'une autre, notamment pour attirer l'attention de son public :

Seigneur qui Dieu devez ameir [...]

A vos toz fas je ma clamour

D'Ypocrisie $[\ldots]^{27}$.

$\mathrm{Ne}$ covient pas je vos raconte

Coument je me sui mis a hunte,

Quar bien aveiz oï le conte

25 Daniel Poirion, «Écriture et ré-écriture au Moyen Âge », Littérature, vol. 41, Intertextualité et roman en France, au Moyen Âge, 1981, p. 114

${ }^{26}$ Parmi onze propositions, dix portent du moins un indicateur de la deuxième personne du pluriel : soit apostrophe, soit pronom personnel, soit verbe conjugué, soit possessif. La seule exception est le topos saisonnier du v. 2.

27 D'Hypocrisie, v. 1 et 6-7, dans MZ, éd. cit., p. 136. 
En queil meniere

Je pris ma fame darreniere,

Qui bele ne gente nen iere ${ }^{28}$.

Si l'on se tourne vers ses prédécesseurs ou contemporains, outre cet emploi ordinaire, on trouve des cas intéressants, tels que les Vers de la Mort d'Hélinand de Froidmond où le poète prend la mort pour son messager auprès de ses amis ${ }^{29}$, ou les Congés d'Arras dont la plupart des strophes sont adressées à différents destinataires, soit directement, soit, comme chez Hélinand, par l'intermédiaire d'une abstraction personnifié $^{30}$. Le lyrisme courtois s'adresse lui aussi souvent à un $t u$ ou un vos, non seulement dans l'envoi, mais aussi dans le corps même de la chanson, par le biais de l'apostrophe exclamative à la dame absente, afin d'accentuer les sentiments du $j e^{31}$.

Sans prétendre à l'exhaustivité, cette typologie montre qu'aucun de ces usages ne s'adapte sans faille au cas des Ribauds de Grève. L'adresse aux vos ne se réduit pas en l'occurrence à un effet rhétorique, qu'il s'agisse d'exprimer l'émotion du moi ou de stimuler l'intérêt du récepteur. Le poète se tourne vers les ribauds comme si ces derniers étaient présents sur le même plan que lui, et comme si son discours atteignait directement leurs oreilles. À cette situation de dialogue se

${ }_{28}$ Complainte Rutebeuf, v. 1-6, dans MZ, éd. cit., p. 138.

29 «Morz, va m'a çaus qui d'amors chantent / Et qui de vanité se vantent, / Si les apren si a chanter [...] » (Hélinand de Froidmont, Les Vers de la Mort, str. II, v. 1-3, éd. Fredrik Wulff et Emmanuel Walberg, trad. Michel Boyer et Monique Santucci, Paris, Champion, 1983, p. 60).

30 «Congié demand tout premerain / A celui qui plus m'est a main / Et dont je miex loer me doi :/ Jehan Boschet, a Dieu remain!»; «Joie, qui vers moi es repointe, / Dusqu'a Biaumés fai une pointe;/ Si me salue a cuer haitié / Le chastelain [...]» (Jean Bodel, Congés, v. 13-16 et 121-124, dans Les Congés d'Arras, éd. Pierre Ruelle, Bruxelles/Paris, Presses universitaires de Bruxelles/PUF, 1965 , p. 85 et 89 ).

31 «Merchi, dame, la cui biautés sourvaint / Mon cuer, qui vous a fait loial eommage !»: Adam de La Halle, Chanson IV, v. 33-34, dans Euvres complètes, éd. et trad. Pierre-Yves Badel, Paris, Le Livre de Poche, 1995, p. 48. Voir Roger Dragonetti, La Technique poétique des trouvères dans la chanson courtoise, op. cit., p. 278-286 et 304-378. 
mêlent inextricablement des éléments descriptifs, qui valent alors comme autant d'insultes. S'y ajoutent les adverbes temporel or (v. 1, 5 et 12) et d'intensité si (v. 7 et 8), contribuant à créer un fort effet de présence. La figure d'hypotypose, qui normalement consisterait en description vivante d'un objet extérieur, arrive là à sa limite pour s'approcher du domaine du théâtre, domaine par excellence du dialogue et du temps présent, dans lequel il ne s'agit pas seulement de représenter une scène, mais de monter sur la scène, de la vivre ${ }^{32}$.

Certes, il n'y a dans ces douze vers aucun dialogue à proprement parler. Le poète monopolise la parole, ses partenaires demeurent complètement muets. Mais dans la logique de ce quasi- ou pseudothéâtre, ces derniers sont supposés proches de l'énonciateur dont le discours doit être entendu directement par eux. C'est pourquoi ce poème ne peut être qualifié de monologue, celui-ci étant, pour reprendre la définition de Catherine Kerbrat-Orecchioni, « un discours que L [= locuteur ou émetteur] n'adresse apparemment à personne d'autre que lui-même $\mathrm{e}^{33} »$. Au contraire, notre scène veut que, auprès de celui qui parle, existent bien ceux qui l'écoutent. Selon la terminologie de la linguiste, il s'agit plutôt d'un «"discours monologal", adressé à un allocutaire déterminé, sans qu'il en soit attendu ni sollicité de réponse, c'est-à-dire sans qu'il y ait "dialogue" à proprement parler », et qui se distingue ainsi du «monologue non adressé », ou

\footnotetext{
32 On sait bien que la théâtralité est un des traits constitutifs de la littérature médiévale en général, au point que, pour citer Paul Zumthor, «toute poésie y participait plus ou moins à ce que nous nommons théâtre », contrairement à la situation moderne où « le théâtre est un art que seul un abus de langage permet de classer parmi les genres littéraires » (Essai de poétique médiévale, op. cit., p. 509). Pour une approche un peu plus nuancée de ce genre en germe à l'époque de Rutebeuf, voir Silvère Menegaldo, «Les jongleurs et le théâtre en France au $\mathrm{XIII}^{\mathrm{e}}$ siècle. Leurs activités et leur répertoire », Romania, vol. 128, 2010, p. 46-91.

${ }^{33}$ Catherine Kerbrat-Orecchioni, «Pour une approche pragmatique du dialogue théâtral », Pratiques, vol. 41, 1984, p. 46-62, ici p. 54.
} 
« soliloque ${ }^{34} »$.

De plus, sans être un dialogue, l'énoncé du poète est toujours susceptible d'être suivi par une réponse que les ribauds, ou du moins l'un entre eux ${ }^{35}$, pourraient y donner, en prenant à leur tour la place de la première personne. Car, selon Émile Benveniste, « "je" et "tu" sont inversibles : celui que "je" définis [sic.] par "tu" se pense et peut s'inverser en "je", et "je" (moi) devient un "tu"36 ». Bien que virtuelle, cette réversibilité n'en est pas moins constitutive de la structure des Ribaud de Grève.

Il existe par ailleurs un grand nombre de textes où cette réversibilité de je et de $t u$ est réalisée d'une manière ou d'une autre et donne lieu à un vrai échange de paroles. D'abord, chez Rutebeuf luimême, il y a deux Disputaisons, l'une de Charlot et du Barbier de Melun, et l'autre du croisé et du décroisé. Toutes les deux sont composées de plusieurs huitains, à la différence des Ribauds de Grève, constitués d'un seul douzain. Cependant, ces disputaisons et ce dit ont en commun d'adopter le schéma de rimes croisées. De plus, dans la dispute entre Charlot et le Barbier, deux jongleurs rivaux, on remarque que les discours directs contiennent chaque fois une apostrophe à l'autre, ou aux autres, quand Rutebeuf y intervient et interpelle les deux compagnons à la fois. Le discours s'ouvre le plus souvent par ces apostrophes :

\footnotetext{
34 Ibid., n. 10.

35 Nous avons du moins un texte médiéval où, représentant plusieurs allocutaires, l'un d'entre eux prend la parole pour répondre au locuteur initial : c'est Aucassin et Nicolette. Quand Nicolette, puis Aucassin, rencontrent des pastoureaux, l'une comme l'autre s'adressent à ceux-ci en les appelant «Bel enfant» au pluriel; et c'est toujours «li uns qui plus fu enparlés des autres » qui leur donne la réplique (éd. et trad. Marie-Françoise Notz-Grob, dans Nouvelles courtoises occitanes et françaises, Paris, Le Livre de Poche, 1997, p. 664-666 et 670-672).

${ }_{36}$ Émile Benveniste, «Structures des relations de personne dans le verbe », dans Problèmes de linguistique générale, I, Paris, Gallimard, 1966, p. 225-236, ici p. 230.
} 
« Charlot, tu vas en compaignie

Por crestientei desouvoir. »[...]

«Barbier, foi que doi la banlive

Ou vos aveiz votre repaire,

Vous aveiz une goute vive. » $[\ldots]$

«Seigneur, par la foi que vos doi, Je ne sai le meillor eslire ${ }^{37}$.»

Pourtant, toutes différences formelles mises à part, la concordance n'est pas parfaite sur le fond non plus : tiers et observateur, le poète ne se compromet pas lui-même dans les débats en tant qu'une des parties, même s'il intervient comme arbitre à la fin de la Disputaison de Charlot et du Barbier de Melun. La nature de ces textes se révèle aussi, sinon plus, narrative que théâtrale, les répliques restant encadrées par le discours direct rapporté ${ }^{38}$.

On peut encore proposer une comparaison avec une autre pièce : elle n'est pas de Rutebeuf, mais appartient à une période d'écriture quasi-contemporaine; elle met en scène elle aussi des personnages ribauds, qui sont cette fois bien définis par leur métier, celui de jongleur, comme l'étaient Charlot et Barbier. Il s'agit de Deus bordeors

37 Disputaison de Charlot et du Barbier de Melun, v. 11-12, 17-19 et 89-90, dans MZ, éd. cit., p. 783 et 790. Quant à la Disputaison du croisé et du décroisé, l'usage du vocatif y est beaucoup moins régulier. C'est peut-être parce que leur enjeu est de discuter sur un sujet précis - si l'on doit prendre la croix ou non - non pas de s'insulter mutuellement. Le croisé comme le décroisé ne font que représenter chacun une position, sans recevoir une identité concrétisée.

${ }_{38}$ À propos de certains textes satiriques du XVI siècle, Jean-Claude Aubailly propose la formulation suivante, qui semble convenir à nos textes composés trois siècles plus tôt : «lorsque le conteur-chansonnier sacrifie à la satire sociale, qu'il lise ou qu'il récite, il semble avoir éprouvé le besoin de s'identifier à un personnage, de faire voir par les yeux d'un tiers pour décupler la force de sa satire ou simplement la rendre plus vivante. Il fait donc appel à des procédés de caractère dramatique, mais sans abandonner totalement les caractéristiques qui font de lui un récitant autant qu'un acteur, c'est-à-dire, de temps à autre, une certaine "distanciation" par rapport à son récit. Ainsi ces pièces peuvent-elles être considérées comme participant à la fois du genre narratif et du genre dramatique » (Jean-Claude Aubailly, Le Monologue, le dialogue et la sottie. Essai sur quelques genres dramatiques de la fin du Moyen Âge et du début du XVI siècle, Paris, Champion, 1976, p. 25). 
ribauz, ensemble de trois monologues dramatiques constitutifs d'un débat, dont chacun présente une tirade mêlée de vantardises et d'invectives que se lancent l'un à l'autre deux bourdeurs ${ }^{39}$. Sur le plan formel, ces pièces peuvent paraître n'avoir rien de commun avec le dit de Rutebeuf: en rimes plates, chacune d'elles est sensiblement plus longue que ce dernier (en comptant entre 164 et 182 vers). Certaines parentés méritent toutefois d'être considérées. À première vue, on retrouve des traits semblables à ce que l'on voit dans les Ribauds de Grève, par exemple la qualité de vêtements et de souliers tournée en dérision :

Tu ne sez vaillant deus festuz !

Com tu es ore bien vestuz

De ton gaaignaige d'oan!

Voiz queus sollers de cordoan

Et com bone chauces de Bruges!

Certes, ce n'est mie de druges

Que tu es si chaitis et las ${ }^{40}$.

Mais ce qui est plus intéressant et plus délicat par rapport à notre propos touche la tradition manuscrite. Car aucun témoin ne possède l'ensemble des trois parties en même temps: un manuscrit du $\mathrm{XIII}^{\mathrm{e}}$ siècle contient le premier et le deuxième discours (intitulés respectivement De deus bordeors ribauz et La response de l'un des

\footnotetext{
39 Voir la notice à l'édition de cette œuvre dans Le Jongleur par lui-même. Choix de dits et de fabliaux, trad. Willem Noomen, Louvain/Paris, Peeters, 2003, p. 25-27, où on lit : "L'ensemble doit dater de la fin du $13^{\mathrm{e}}$ siècle [...] Il est assez probable qu'il s'agit d'œuvres d'Adenet le Roi, dont les années d'activité littéraire s'étendent de 1269 à 1285 ; les pièces des Deus bordeors ribauz n'auront donc pas été composées avant les années soixante-dix» (p. 27), et l'introduction d'Edmond Faral à son édition du même texte : Mimes français du XIII siècle (textes, notices et glossaire), éd. Edmond Faral, Paris, Champion, 1910, p. 83-91. Pour distinguer les trois parties de cette œuvre composite, nous adoptons la numérotation adoptée par ces deux éditions.

${ }^{40}$ De deus bordeors ribauz, pièce I, v. 7-13, dans Le Jongleur par lui-même, éd. Willem Noomen, éd. cit., p. 28.
} 
deus ribauz $)^{41}$; un autre de la même époque, le premier (La gengle au ribaut) et le troisième (La contregengle $)^{42}$. Mais il en existe encore un autre du début du XIV ${ }^{\mathrm{e}}$ siècle qui, curieusement, ne présente que la partie initiale, assortie d'un titre qui lui est propre : Li esbaubisemanz lecheor ${ }^{43}$. Cette dispersion s'explique par la logique même de cet assemblage basé sur un jeu d'échanges. Que les deux dernières pièces, composées visiblement par différents auteurs à différents desseins, apparaissent chacune comme une réaction à la première, cela les empêche de coexister sur le même plan, sous peine de s'invalider l'une l'autre.

En ce qui concerne la genèse de cet étrange ensemble, Edmond Faral, suivi par Willem Noomen, penche pour l'hypothèse selon laquelle un même auteur aurait rédigé les deux premiers textes comme un débat à deux voix, tandis que le troisième se serait substitué au deuxième à l'initiative d'un autre ménestrel. Mais il n'est pas exclu que la version originelle n'ait connu que la première partie et que chacune des deux réponses y ait été ajoutée respectivement et indépendamment par deux auteurs distincts. De toute façon, nous saisissons là un jeu dialogique dont le dynamisme est à la fois très marqué et très souple, si bien que le discours initial appelle une réponse, susceptible de varier selon le sens et l'effet souhaités, tandis que le premier propos pouvait se tenir seul, isolément, tout en gardant sa

\footnotetext{
${ }^{41}$ Paris, BnF, ms. fr. 19152, fol. 69v-70v. Voir la notice de ce manuscrit dans la base de Jonas, site internet: http://jonas.irht.cnrs.fr/manuscrit/45865 (page consultée le 26 août 2015).

42 Paris, BnF, ms. fr. 837, fol. 213v-215r. Voir la notice dans la base de Jonas, site internet : http://jonas.irht.cnrs.fr/manuscrit/45562 (page consultée le 26 août 2015).

${ }^{43}$ Berne, Bibliothèque de la Bourgeoisie, 354, fol. 65v-67r. Voir la notice dans la base de Jonas, site internet : http://jonas.irht.cnrs.fr/manuscrit/8202 (page consultée le 26 août 2015). Nous ne prenons pas en compte le ms. Paris, BnF, ms. fr. 12483 (fol. 238v) qui ne retient que les six premiers vers de la première pièce comme une citation.
} 
capacité d'ouvrir un dialogue.

Revenons sur notre poème, à propos duquel se formulerait ainsi notre question: ce même type de jeu dialogique se trouverait-il au fondement des Ribauds de Grève ? Il n'est pas question d'en imaginer quelque réplique qui ne soit pas parvenue jusqu'à nous, mais de se figurer l'image elle-même que le poète voulait susciter dans l'esprit de son public, et qui est d'une importance capitale pour l'efficacité de sa parole. Il est alors évident que dans ce dit, la présence des ribauds est trop marquée pour être reléguée en arrière-plan comme simple élément décoratif, et que c'est cette présence des allocutaires qui donne son sel au croquis laconique.

La chose, au demeurant, n'est guère originale, dès lors que la formalisation littéraire de l'échange de paroles était tellement pratiquée au Moyen Âge que l'on peut en dire qu'elle a fait naître un genre, le débat ${ }^{44}$. Par ailleurs, ce mécanisme du dialogue ressortit depuis toujours aux universaux du langage humain, comme l'affirme Émile Benveniste :

[...] je pose une autre personne, celle qui, tout extérieure qu'elle est à «moi », devient mon écho auquel je dis $t u$ et qui me dit $t u$. La polarité des personnes, telle est dans le langage la condition fondamentale, dont le procès de communication [...] n'est qu'une conséquence toute pragmatique ${ }^{45}$.

Mais une même condition initiale ne conduit pas toujours à une même conséquence finale. Pour diverses raisons, la communication

\footnotetext{
44 De cette catégorie de textes, Pierre-Yves Badel offre une approche globale, mais qui porte principalement sur les derniers siècles du Moyen Âge : Pierre-Yves Badel, «Le débat », dans Grundriss der Romanischen Literaturen des Mittelalters, t. 8/1, La Littérature française aux XIV et $X V^{e}$ siècles (Partie historique), dir. Daniel Poirion, Heidelberg, Carl Winter Universitätsverlang, 1988, p. 95-111. L'étude de Silvère Menegaldo rend compte de son existence au XIII ${ }^{\mathrm{e}}$ siècle en fonction du rapport avec la pratique théâtrale des jongleurs ( Les jongleurs et le théâtre en France au XIII ${ }^{\mathrm{e}}$ siècle », art. cit., p. 69-77).

45 Émile Benveniste, «De la subjectivité dans le langage », dans Problèmes de linguistique générale I, op. cit., p. 258-266, ici p. 260.
} 
effective risque d'échouer. C'est le cas des chansons courtoises qui se substituent souvent à un dialogue réel, devenu impossible soit par la perfidie des losengiers, soit à cause de la peur qu'a l'amant d'être refusé par celle qu'il désire. Mais l'incommunicabilité se révèle plus fondamentale lorsque l'on franchit la frontière de son milieu socioculturel pour rencontrer quelqu'un de condition différente. Comme l'a récemment démontré Michel Zink, un bon nombre de textes médiévaux mettent en scène l'embarras du dialogue avec les vilains ou simples, embarras qui tient à la disparité des codes ${ }^{46}$. Telle est aussi à peu près la situation que Rutebeuf rapporte dans une strophe de son dit De sainte Église :

Je ne blame pas gent menue :

Il sont ausi com beste mue ;

L'en lor fet canc' on veut acroire,

L'en lor fet croire de venue,

Une si grant descovenue

Que brebiz blanche est tote noire.

« Gloria laus », c'est « gloire loire »;

Il nous font une grant estoire

Nes dou manche de la charrue,

Pour coi il n'ont autre mimoire.

Dites lor «c'est de saint Gregoire »,

Quelque chose soit est creüe ${ }^{47}$.

Le $j e$ de ce poème parle en tant que clerc, et la citation de

\footnotetext{
${ }^{46}$ Michel Zink, « Parler aux "simples gens". Un art littéraire médiéval », cours de la chaire Littératures de la France médiévale au Collège de France du 10 décembre 2014 au 18 février 2015, en particulier la troisième séance du 7 janvier 2015, site internet : $\quad$ http://www.college-de-france.fr/site/michel-zink/course-2015-01-0710h30.htm (page consultée le 26 août 2015). Parmi les exemples qu'il analyse se trouve notamment l'épisode de la rencontre de Calogrenant avec le gardien de taureaux au début du Chevalier au Lion: Chrétien de Troyes, Le Chevalier au Lion, v. 276-407, éd. et trad. David F. Hult, Paris, Le Livre de Poche, p. 66-74.

47 De sainte Église, v. 97-108, dans MZ, éd. cit., p. 190. Le mot loire au v. 103, substituant dans les oreilles des illettrés au latin laus «louange », peut signifier « soit la loutre, soit la cuve du pressoir ou le vin sortant du pressoir »: ibid., p. 190-191; voir aussi CEuvres complètes de Rutebeuf, éd. Edmond Faral et Julia Bastin, éd. cit., t. 1, p. 283.
} 
l'hymne latin témoigne en soi de ce statut honorable. Mais son privilège d'orator n'impressionne pas beaucoup la gent menue. Ces êtres sans raison ne peuvent pas comprendre son langage grandiose, signe par excellence de la gloria cléricale, qui, de ce fait, finit par se réduire à quelque bruit insignifiant. En fait, ce blâme par prétérition n'a pas pour auditeur ce bas peuple, qui ne constitue qu'un exemple que ne doivent pas suivre les sages. Il y a là évidemment un sentiment de supériorité à l'égard des simples, sentiment partagé par tout le clergé. Mais cela ne parvient pas à gommer la confusion qu'aurait ressentie ce lettré devant la masse indifférente et impénétrable. D'autant moins que nous sommes en train de lire un pamphlet lancé contre les ordres mendiants: ces vers reflètent la rivalité qui oppose les clercs traditionnels - y compris les maîtres séculiers de l'Université pour lesquels se bat Rutebeuf - aux frères dominicains et franciscains, spécialistes de la «parole nouvelle ${ }^{48}$ » et capables en tant que tels d'attirer plus facilement l'attention de la population dépourvue de raffinement intellectuel. Par l'incompétence des ignorants qu'il veut ridiculiser, Rutebeuf fait entrevoir, malgré lui, l'impuissance du langage du savoir.

Ce constat général de la difficulté à «parler aux "simples

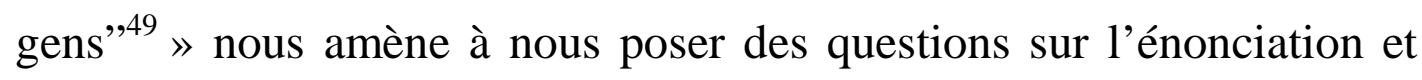
ses circonstances dans les Ribauds de Grève, parce que contrairement à l'apparence, la communication avec ces ribauds, probablement aussi simples que cette gent menue-là, n'a rien d'évident. En d'autres termes, pour vraiment parler avec eux, pour se faire comprendre d'eux, il faut

\footnotetext{
48 Jacques Le Goff et Jean-Claude Schmitt, «Au XIII siècle : une parole nouvelle », dans Histoire vécue du peuple chrétien, dir. Jean Delumeau, 2 vol., Toulouse, Privat, 1979, t. 1, p. 257-279.

49 Nous reprenons l'expression qu'a donnée Michel Zink au titre de son cours au Collège de France. Voir supra, n. 46.
} 
que le je adopte une certaine manière de discourir et revête une persona particulière qui permettrait de réduire l'écart social entre lui-même et ses allocutaires. Nos questions se résumeraient donc à celle-ci : qui parle aux ribauds, et comment?

\section{Je suis là où je parle}

Si le je des Ribauds de Grève semble à l'évidence représenter le poète lui-même, cette assimilation ne dit rien d'utile sur son identité poétique qui reste à déterminer en fonction de ce qui se passe dans le texte, entre lui et ceux auxquels il s'adresse. Or, si l'on suit jusqu'au bout la logique que le poème impose, il est inconcevable, même sans réaction, que le propos du je ne soit pas pleinement entendu par les vos. Car, tout simplement, une moquerie de ce type ne fonctionne que si elle est reçue et comprise par ceux qu'elle vise. Rutebeuf n'est donc pas ici dans la même posture que pour le dénigrement des illettrés dans $D e$ sainte Église, pas plus que son langage n'est le même dans les deux cas. S'adressant aux ribauds, il doit adopter un ton accessible à ces simples gens, et utiliser des mots qu'ils peuvent déchiffrer et, le cas échéant, employer.

L'accessibilité de ce langage pour les ribauds, la Griesche d'hiver vient la prouver. Dans ce poème de l'infortune à plein droit, le je prend un rôle et une voix qui sont censés être d'un pauvre démuni, sans doute débauché, inintelligent en apparence, à savoir un ribaud. Mais, homme de «povre sens et povre memoire ${ }^{50}$ », il n'en peut pas moins exploiter un riche territoire poétique, territoire rutebovin par excellence aux yeux des lecteurs modernes, recouvrant à partir des motifs de la froidure et de la nudité jusqu'à la figure imagée des mouches blanches, bref tout ce

${ }^{50}$ Griesche d'hiver, v. 10, dans MZ, éd. cit., p. 196. 
qu'on lit de façon plus condensée dans les Ribauds de Grève. Partant, nous pouvons faire l'hypothèse que le sarcasme contre les ribauds ressortit d'un code qui ne leur est pas étranger, ce que confirme définitivement la coda de la Griesche d'hiver:

Cil qui devant cousin le claime

Li dist en riant : « Ci faut traime

Par lecherie.

Foi que tu doiz sainte Marie,

Car vai or en la draperie

Dou drap acroire.

Se li drapiers ne t'en wet croire,

Si t'en revai droit à la foire

Et vai au Change.

Se tu jures saint Michiel l'ange

Qu'il n'at sor toi ne lin ne lange

Ou ait argent,

Hon te verrat moult biau sergent,

Bien t'aparsoveront la gent :

Creüz seras.

Quant d'ilecques te partiras,

Argent ou faille enporteras $»^{51}$.

Avec des différences de détail et une ironie plus sournoise, on retrouve ici la même situation de fond que dans les Ribauds de Grève. Cet ancien cousin n'a d'autre envie que de tourner en dérision ce biau sergent $^{52}$, de même que Rutebeuf ne pensait pas à aider les ribauds $a$ point. Mais dans cet exemple, on voit clairement le fait qu'un tel pauvre fou saisit bien, au-delà de toute antiphrase, le malin sous-entendu de ce

\footnotetext{
51 Griesche d'hiver, v. 88-104, dans MZ, éd. cit., p. 200 et 202. Il convient de noter que, dans le dernier quart de cette poésie personnelle, Rutebeuf substitue la troisième personne à la première, et déploie une observation générale du type du joueur fou : «Li dei m'ont pris et empeschié : / Je les claim quite ! / Foux est qu'a lor consoil abite [...]» (ibid., v. 74-76, p. 200). Après l'énoncé du cousin, le je reprend la parole pour les trois derniers vers du texte que nous allons citer.

${ }^{52}$ C'est en effet une variante pittoresque du constat général des Plaies du monde: « N'i vaut riens parenz ne parente : / Povre parent nuns n'aparente, / Mout est parens et pou amis. / Nuns n'at parens c'il n'i a mis /[...] / Qui auques at, si est ameiz, / Et qui n'at riens c'est fox clameiz » (v. 13-16 et 21-22, dans MZ, éd. cit., p. 72).
} 
que « li dist en riant » son faux parent, car il va en conclure :

Or ai ma paie.

Ensi chascuns vers moi s'espaie, Si n'en puis mais ${ }^{53}$.

Face au railleur, sa victime ne fait aucune riposte ; elle semble résignée à sa paie désagréable. Mais l'affaire n'en reste pas là. Quand un fait langagier revêt une forme textuelle, il ne se clôt pas sur la relation initiale entre le locuteur et l'allocutaire, mais se transpose en un dynamisme trilatéral de sorte qu'il faut désormais compter avec un troisième pôle, celui du lecteur, ou de l'auditeur, dernière instance à même de neutraliser le rapport de forces établi dans la diégèse ${ }^{54}$. Incapable de se venger lui-même, le poète peut du moins exposer le taquin au rire, voire au reproche de ce tiers, quitte à s'y exposer de nouveau lui-même.

Mais nos Ribauds de Grève n'écriront aucun poème et resteront à jamais silencieux. À la différence de la Griesche d'hiver, ce poème n'appartient pas aux offensés, mais à l'offenseur, qui veut en tirer parti en croyant que sa joyeuse maltraitance de ces gueux va plaire à la clientèle de sa rime. Et vu la qualité du produit, son succès est très probable ; l'auditeur, ou lecteur, doit en rire aux éclats. Pourtant ce succès est ambigu, de même que ce rire qui s'arrête à peine aux seules victimes dont le poète s'est déjà moqué. Ainsi, du piège que lui-même a tendu aux ribauds, pas plus que ceux-ci, ce malin ne sort pas indemne, voire triomphant. C'est que la mise en scène des vos interdit en elle-

\footnotetext{
53 Griesche d'hiver, v. 105-107, dans MZ, éd. cit., p. 202.

54 Très suggestif à cet égard est le travail de Catherine Kerbrat-Orecchioni («Pour une approche pragmatique du dialogue théâtral », art. cit.) : il consiste à analyser le fonctionnement de diverses formes de dialogue théâtral en prenant en compte les déviations par rapport à la conversation ordinaire, provoquées par la présence du public. Nous tenons à rappeler encore une fois que la théâtralité était un support indispensable pour l'existence de la littérature au Moyen Âge et, en particulier, pour celle des textes classables dans la catégorie du dit, dont relevait la plus grande partie de l'œuvre de Rutebeuf.
} 
même l'effacement du je. Tant qu'il dit vos, le je s'associe obligatoirement à ceux à qu'il parle. Tant que les vos ne se découvrent pour les autres qu'à travers sa parole, le je n'échappe pas non plus à leur regard : ce regard de spectateur tellement avide d'objets de risée qu'il met dans un même sac tous ceux qu'il surprend sur la scène, les moqués et le moqueur à la fois, pour se moquer à son tour des uns comme de l'autre. Tant pis pour le je. Car, même quand il ne souffle pas un seul mot sur lui-même, ni un seul pronom de la première personne, c'est déjà le fait de l'énonciation, le dit, qui finit par accuser sa présence, et cela tout près des vos.

Dès lors, ce dont le je parle aux vos se retourne contre lui, par la proximité qu'il crée entre eux et lui-même, proximité fondée sur son langage qui leur est accessible au sens plein du terme, physiquement et intellectuellement, ce qui impose à l'un et aux autres quelque destin commun : si les ribauds se trouvent sur la place de Grève ${ }^{55}$, là est aussi

55 Edmond Faral remarque que ce toponyme, absent dans le corps du texte, ne se trouve que dans le titre donné par le seul témoin du poème : Paris, BnF, ms. fr. 1635, fol. $44 \mathrm{v}$, tout en rappelant une autre esquisse des ribauds de la même place, dessinée cette fois par Jean de Meun : «maint ribaut ont les queurs si bauz, / portanz sas de charbon an Greve, / que la peine riens ne leur greve, / s'il en pacience travaillent, / qu'il balent et tripent et saillent / et vont a Seint Marcel aus tripes / ne ne prisent tresors .III. pipes, / ainz despendent en la taverne / tout leur gaaign et leur esperne, / puis revont porter les fardeaus / par leesces, non pas par deaus, / et leaument leur pein gaaignent / quant enbler ne tolir ne daignent, / puis revont au tonel et boivent / vivent si con vivre doivent » (Le Roman de la Rose, v. 5018-5032, éd. Félix Lecoy, Paris, Champion, 1965-1970, 3 vol., t. 1, p. 155 ; Euvres complètes de Rutebeuf, éd. Edmond Faral et Julia Bastin, éd. cit, t. 1, p. 531). Alain Corbellari, de son côté, oppose le fatalisme des marginaux dans le poème de Rutebeuf à la vigueur que reconnaît le continuateur du Roman de la Rose aux travailleurs manuels, en même temps qu'il note lui aussi chez Rutebeuf l'absence de localisation explicite, qu'il pense pouvoir imputer à une certaine intériorisation de la topographie parisienne (La Voix des clercs, op. cit., p. 161-165). Quant à la précision du lieu dans le titre du dit, il ne serait pas exclu qu'elle ait été motivée de la part du copiste aussi bien par le souvenir du Roman de la Rose que par la scène quotidienne sur le rivage de la Seine, en rapportant ainsi un effet de réel à un effet $d u$ réel, pour reprendre deux notions distinguées par Nancy Freeman Regalado: la première, qu'elle s'approprie de Roland Barthes, renvoie à la «representation» en tant que «our imaginative construction », telle 
le poète ; s'ils sont agacés par les mouches blanches, elles doivent tout autant tomber sur sa tête ; s'ils sont maltraités et dépouillés par le jeu de Fortune, il faut que lui-même, dans sa sécurité momentanée, prenne tout de même garde, puisque la roue de cette déesse :

[...] torne en petit d'eure, Que li serviauz chiet en la boe

Et li servant li corent seure :

Nus n'atant a leveir la poe.

En cort terme a non Chantepleure ${ }^{56}$.

Dans ce jeu de proximité et de promiscuité, la «distance de la dérision $^{57} \gg$ ne s'établit pas tant entre les ribauds et le poète qu'entre ceux-là et celui-ci d'un côté, et le public de l'autre. Une fois de plus, la situation est comparable à celle des Deus bordeors ribauz, qui rivalisent dans le dénigrement mutuel afin de se démarquer l'un de l'autre, alors que les auditeurs, auxquels le dernier mot est confie $^{58}$, doivent leur renvoyer équitablement leur rire, à moins que le prix ne revienne au plus ridicule.

qu'elle est suscitée par «words in text», tandis que la seconde, de son cru, à la "reference, which describes the relation between representation and what exists in the historical world» (Nancy Freeman Regalado, «Effet de réel, Effet du réel: Representation and reference in Villon's Testament », Yale French Studies, vol. 70, 1986, p. 63-77, ici p. 64 ; voir aussi Roland Barthes, «L'effet de réel », dans Littérature et réalité, dir. Gérard Genette et Tzvetan Todorov, Paris, Le Seuil, 1982 [1968], p. 81-90). Ajoutons encore qu'en deçà de la différence de tempérament ou de conception du monde des auteurs, les textes de Rutebeuf et de Jean de Meun divergent d'abord dans leur manière et leur perspective : proximité dialogique d'un côté ; distance descriptive, voire moralisatrice de l'autre.

${ }_{57}$ De monseigneur Ancel de l'Isle, v. 36-40, dans MZ, éd. cit., p. 98.

57 «La figure du poète brisé par les chaos de la vie s'impose en offrant sa faiblesse au rire. Le comique n'est donc pas présent accidentellement dans cette poésie. Il est la voie choisie pour donner au je sa consistance et dissuader le public de se l'approprier, comme la chanson lui avait donné l'habitude de le faire »: Michel Zink, La Subjectivité littéraire. Autour du siècle de saint Louis, Paris, PUF, 1985 , p. 67.

${ }^{58}$ C'est ainsi que se termine le deuxième morceau du dialogue, la Response de l'un des deus ribauz: " Beaus seignor, vos qui estes ci, / Qui noz parole avez oï, / Se j'ai auques mielz dit de li, / A toz ge vos requier et pri / Que le metez fors de ceanz, / Que bien pert que c'est un noienz ! : De deus bordeors ribauz, pièce II, v. 159164, dans Le Jongleur par lui-même, éd. Willem Noomen, éd. cit., p. 52). 
De cela, Rutebeuf est-il conscient? Sans aucun doute. C'est sur la même esthétique de la présence, c'est-à-dire au risque d'être bafoué, que se fondent ses poésies personnelles. Cette règle de jeu, il l'accepte presque ostensiblement, non sans amertume néanmoins :

L'an dit que fox qui ne foloie pert sa saison :

Que je n'ai borde ne maison, Suis je mariez sans raison ${ }^{59}$ ?

Un autre point commun à remarquer entre le poème aux vos et ceux du je, c'est une forte prise de conscience de l'effet de la voix. Cette conscience se traduit de façons diverses, mais toujours avec une même intensité. Ainsi, d'un côté, les vers des Ribauds de Grève sont dominés par la rapidité et la brièveté qui ne permettent guère - à une seule exception près (aux vers 5-6) - que chaque proposition dépasse la limite d'un octosyllabe ; ils imitent de cette manière la violence du coup du vent tel qu'il vente, sous les yeux du poète, sur la place publique découverte. De l'autre, certains poèmes d'effusion sont marqués par le rythme saccadé du tercet coué, reproduisant en quelque sorte le claquement de dents de celui qui est soumis à l'influence du froid $^{60}$. Dans tous les cas, la présence du je s'affirme d'abord par et dans ses actions verbales. C'est ce que font entendre li vers changié à l'approche de l'hiver :

\footnotetext{
59 Mariage Rutebeuf, v. 21-24, dans MZ, éd. cit., p. 68.

${ }^{60}$ Voir supra la citation de la Griesche d'été, v. 20-24. Il arrive aussi que le schéma du tercet coué trahisse un chagrin difficilement retenu, quand il est employé dans le registre polémique comme dans D'Hypocrisie ou la Complainte de maître Guillaume de Saint-Amour. De cette «poésie du flot et du flux », Michel Zink explique l'effet en ces termes : «le rythme à la fois satisfaisant et dégingandé du tercet coué, avec la surprise attendue du vers bref qui le termine mais ne le clôt pas, puisqu'il reste sur le suspens d'une rime isolée dans l'attente des octosyllabes du tercet suivant, qui eux-mêmes ont besoin de la chute désinvolte, chantante et lasse du vers de quatre pieds, qui à son tour..., les tercets se poussant et s'épaulant ainsi l'un l'autre comme des vagues, sans pouvoir s'arrêter sinon au prix d'une menue violence métrique » (MZ, éd. cit., p. 33-34).
} 
Contre l'yver,

Dont mout me sont changié li ver

Mon dit commence trop diver

De povre estoire ${ }^{61}$.

Phonétiquement, la saison accapare toute seule trois rimes de suite: yver- $(l) i$ ver-(d)iver, preuve éclatante de la force avec laquelle elle peut s'emparer de l'espace d'un texte. Cependant on s'aperçoit tout de suite que l'exercice de cette force passe par un intermédiaire, celui qui fait son dit, et qui y laisse non seulement des marques de l'influence qu'il subit du monde extérieur, mais aussi et du même coup, des traces de sa propre existence en tant que seul témoin de cette influence imposée. Autrement dit, en lisant un poème hivernal, ce n'est jamais l'hiver en soi que l'on y saisit, mais l'expression de sentiments et de sensations qu'aurait eus le poète «contre l'hiver ». La parole du je se pose comme écran infranchissable devant l'objet dont il parle. Mais cela est non moins vrai pour le sujet : où trouverait-on son portrait sinon dans ce qu'il dit?

Tel dit, tel diseur. L'un et l'autre n'ont qu'un seul et même substrat ontologique en commun, celui du texte. Par conséquent, à chaque fois que se fait entendre une voix, se dessine une silhouette du poète, conformément au ton que cette voix prend et quel que soit le pronom personnel qu'elle émet ${ }^{62}$. Parler à vos converge infiniment vers

${ }^{61}$ Griesche d'hiver, v. 6-9, dans MZ, éd. cit., p. 196. Selon l'éditeur, «divers signifie à la fois "changeant" (le dit est le fruit du changement de saison) et "mauvais" ou "cruel". De plus - le titre et la rime des v. 6-8 le soulignent - ce poème est un dit "d'hiver" » (ibid., p. 198). Plus tard, il développera à partir de ce passage un concept heureux, celui de la «poétique de l'indigence », laquelle produit, selon lui, « un poème misérable sur un sujet misérable »: Michel Zink, «Chanter, dire, conter au Moyen Âge. La poésie comme récit », Cours et travaux du Collège de France. Résumés 2005-2006, 106 année, 2006, p. 763-765.

${ }^{62}$ Nous nous rappelons la Pauvreté Rutebeuf qui finit par un portrait négatif du poète : «Et je n'ai plus que vos veeiz » (v. 48, dans MZ, éd. cit., p. 972). On n’y voit en effet que sa poésie, farcie d'un bout à l'autre de jeux verbaux incongrus qui vident les mots de leur substance, par exemple : «Pou i voi et si i preig pou ; / Il 
parler du moi.

Nous sommes encore loin d'avoir épuisé tous les registres vocaux de Rutebeuf. Il convient d'indiquer du moins, sans entrer dans le détail, l'existence d'une autre voix, non moins fréquente, celle qui parle d'eux, employée surtout dans les poèmes polémiques ou didactiques. Il est vrai que l'on écoute là plutôt un moraliste satirique mais sérieux qu'un jongleur amusant. Nonobstant, on atteste que, même à la troisième personne, son regard erre toujours sur le même endroit que celui où traînent et les misérables ribauds et le pauvre ménestrel, à savoir ce « monde divers » où

Toz fu estez, or est yvers ;

Bon fu, or est d'autre maniere ${ }^{63}$.

Si l'œuvre de Rutebeuf est diverse, si sa voix est changeante et ses rôles, pris les uns après les autres, apparemment contradictoires ${ }^{64}$, ce n'est pas seulement par la faute du poète « qui rudement heuvre ${ }^{65}$ », mais aussi à cause de la diversité du monde où il habite. Mais pour instable qu'il soit en apparence, ce monde, en dernière analyse, reste le même en ceci qu'il s'obstine dans le sens de la perdition. Pareillement, de quelque position qu'il choisisse, Rutebeuf voit et fait voir le même paysage, la même réalité, avec une plus grande acuité pour le mauvais côté que pour le bon. Sa vision se déploie ainsi sous le signe de l'hiver, qui renvoie moins à une période climatique à laquelle succéderait la reverdie printanière qu'à un état $d u$ monde en éternel refroidissement, en perpétuelle déchéance. C'est une telle fixation obsessionnelle sur un

m'i souvient plus de saint Pou / Qu'il ne fait de nul autre apotre » (ibid., v. 41-43). Le dénuement d'un rimeur ne pourrait mieux s'exprimer.

63 État du monde v. 3-5, dans MZ, éd. cit., p. 82. On y retrouve aux v. 3-4 la rime divers - yvers.

${ }^{64}$ L'incohérence flagrante de ces œuvres conduisait autrefois un critique à y voir, non pas un seul auteur, mais plusieurs : voir Edward Billings Ham, «Rutebeuf Pauper and Polemist », Romance Philology, vol. 11, 1957-1958, p. 226-239.

65 Rutebeuf, Mariage Rutebeuf, v. 45, dans MZ, éd. cit., p. 270. 
ici et maintenant, trempée d'un pessimisme incurable, qui soutient l'unité de ce travail poétique mouvant, mais aussi livre un portrait du poète versatile. Et les Ribauds de Grève, ce poème à la deuxième personne fait mettre en perspective, les uns à côté des autres et dans leur ensemble, ceux à la première personne et ceux à la troisième personne, en lançant un pont qui lie l'observation à la compromission. Car, ainsi que nous avons jusqu'ici tenté de le démontrer, celui qui dit vos à son objet a déjà mis un pied, sinon les deux, dans le tableau qu'il tâche de peindre.

En fin de compte, les Ribauds de Grève sont-ils un poème de l'infortune comme les autres? Cette question pourrait paraitre datée depuis qu'en 1989-1990, l'édition de Michel Zink propose une autre façon de lire Rutebeuf, par son refus de toutes classifications en faveur d'un ordonnancement chronologique des textes ${ }^{66}$. Certainement, la nouvelle méthode a ses faiblesses, liées au caractère hypothétique de la datation, et est susceptible de conduire à des lectures plus ou moins douteuses fondées sur la biographie supposée du poète. Mais elle a le mérite, non des moindres, de faire découvrir à la fois la cohérence et la complexité du mouvement d'écriture mené par Rutebeuf; elle rend ainsi sensible au réseau embrouillé que dessine ce mouvement, réseau pour ainsi dire occulté par la nette segmentation des anciennes éditions.

Mais c'est là une autre histoire. En revanche, quand il paraissait inévitable de procéder à un classement thématique ou générique ${ }^{67}$,

66 Cette tentative se base largement sur la chronologie proposée par Michel-Marie Dufeil, «L'œuvre d'une vie rythmée : chronographie de Rutebeuf », dans Musique, littérature et société au Moyen Âge, dir. Danielle Buschinger et André Crépin, Paris, Champion, 1980, p. 279-294.

67 En effet, Edmond Faral et Julia Bastin ont eux aussi estimé que l'ordre chronologique serait le meilleur et que cette manière «aurait fait apparaître l'unité de l'œuvre considérée dans son ensemble en même temps que le processus de son développement: deux préoccupations majeures du travail historique », mais «il 
quelle place assigner à une pièce aussi inclassable que les Ribauds de Grève ? Nous dirions que, en les faisant voisiner avec les poésies dites personnelles, Edmond Faral et Julia Bastin n'avaient pas entièrement tort : nous y entendons clairement une personne parler.

fallait, disaient-ils, y renoncer du moment qu'on n'était pas au clair sur les dates, ni absolues, ni relatives » (Euvres complètes de Rutebeuf, éd. Edmond Faral et Julia Bastin, éd. cit., t. 1, p. 220). Ils ont signalé sans dissimulation le caractère arbitraire du choix final, faute de mieux à leurs yeux, et, comme nous l'avons noté ci-dessus, tenté à l'intérieur de chacune des cinq sections un arrangement chronologique, du moins dans la mesure où les données historiques et parfois littéraires semblaient leur permettre de le faire. Notons par ailleurs que MichelMarie Dufeil reconnaît volontiers sa dette à l'égard de ces philologues dont l'édition «suffit, dit-il, à notre présent bonheur, avec ses longs et parfois judicieux questionnements sur la date de chaque pièce »: Michel-Marie Dufeil, «L'œuvre d'une vie rythmée », art. cit., p. 279. 